\title{
HASTE MRU In the Evaluation of Acute Flank Pain
}

\author{
Jeffrey Mullins, Michelle J. Semins, Mark E. Bohlman, Brian R. Matlaga
}

Departments of Urology and Radiology, The Johns Hopkins University School of Medicine, Baltimore, MD, USA

A 20 year old, otherwise healthy, G2PO, 21 week pregnant female presented to the emergency department with a one day history of left flank pain, nausea, and vomiting. She denied a history of fevers, chills, or dysuria. Physical examination revealed left costovertebral angle tenderness. Laboratory evaluation was significant for leukocytosis $(14,000)$, normal creatinine (0.6), and unremarkable urinalysis. Renal ultrasound demonstrated mild left hydroureteronephrosis without evidence of stone. Subsequent HASTE magnetic resonance urography (MRU) revealed a $3 \mathrm{~mm}$ left ureteral stone, mild hydronephrosis, and a forniceal rupture (Figure-1). The patient was managed conservatively with hydration and oral narcotics.

Urolithiasis is not an uncommon finding in pregnancy with an estimated incidence of $1 / 1,500$

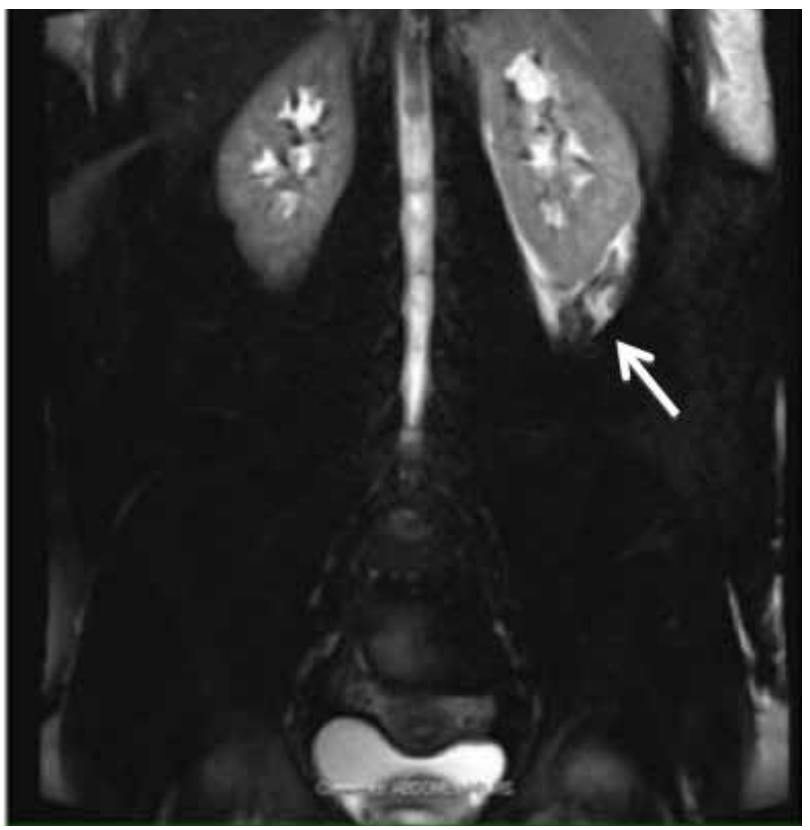

pregnancies (1). Although pregnancy does not confer an increased risk of urolithiasis, a shift in stone profile in pregnant patients has been observed. Specifically, there is an increased incidence of calcium phosphate stones as opposed to calcium oxalate stones most commonly observed in non-pregnant females. Reasons for this shift in stone composition include an absorptive hyercalciuria and relatively alkaline urine $\mathrm{pH}$ that occur in this population (2). Despite the common occurrence of stone disease in pregnancy, safe and accurate diagnosis remains a dilemma.

When subjecting pregnant patients to diagnostic imaging studies the potential impact to the fetus must be considered. Computed tomography (CT), the gold standard for stone diagnosis, exposes the pa-

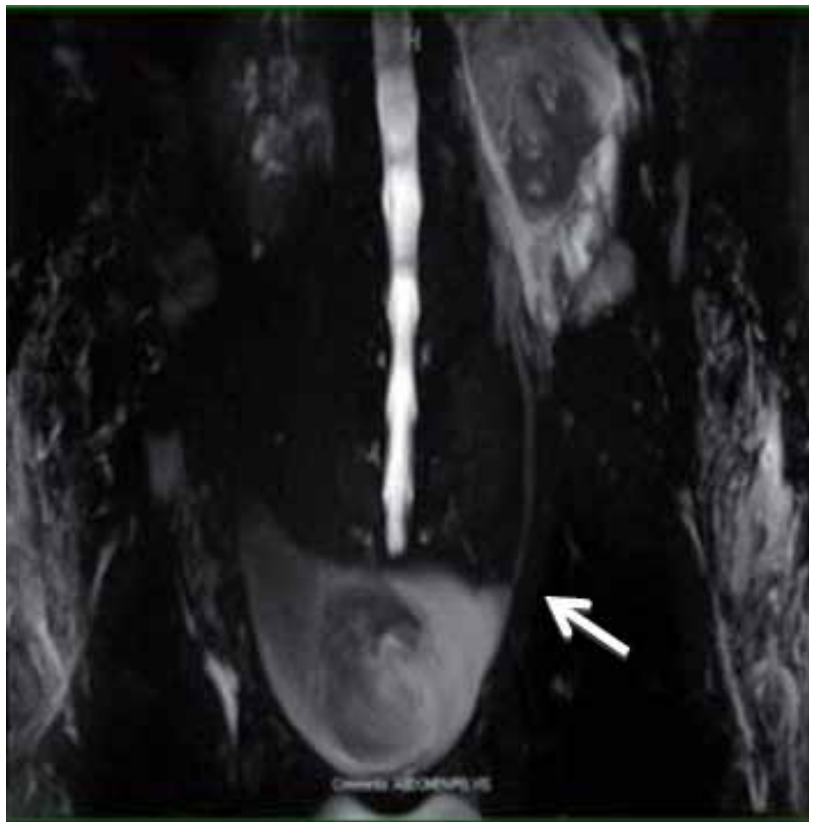

Figure 1 - Magnetic resonance urogram demonstrating A: Forniceal rupture with fluid within Gerota's fascia (arrow). B: Forniceal rupture and fluid within Gerota's fascia again demonstrated as well as a $3 \mathrm{~mm}$ distal ureteral calculus (arrow). 
tient and fetus to ionizing radiation. The amount of radiation exposure to the fetus depends on both the gestational age as well as scanning parameters. On average, a typical pelvic CT scan will expose the fetus to 0.024 Gy in the first trimester and 0.046 Gy in the third trimester (3). While these doses are less than levels which are considered "dangerous", there nonetheless exists concern regarding potential teratogenic and carcinogenic risks to the fetus.

Alternative diagnostic imaging studies imposing no risk to the fetus have been historically limited to ultrasound. This modality, however, has limitations in diagnosing ureteral calculi. HASTE MRU has recently emerged as a safe, highly accurate means of diagnosing ureteral calculi. HASTE MRU utilizes a heavily T2 weighted image that does not require the administration of intravenous

\section{REFERENCES}

1. Meria P, Hadjadj H, Jungers P, Daudon M; Members of the French Urological Association Urolithiasis Committee: Stone formation and pregnancy: pathophysiological insights gained from morphoconstitutional stone analysis. J Urol. 2010; 183: 1412-6.

2. Chen MM, Coakley FV, Kaimal A, Laros RK Jr: Guidelines for computed tomography and magnetic resonance imaging use during pregnancy and lactation. Obstet Gynecol. 2008; 112: 333-40. contrast agents, which are potentially hazardous to the pregnant patient. In a study by Spencer et al., MRU was utilized to evaluate painful hydronephrosis in pregnancy. The authors were able to successfully diagnose all cases of ureteral stones. They also describe the "double kink" sign indicative of distal ureteral obstruction (4). Regan et al. directly compared the ability of spiral CT and MRU to diagnose acute ureteral obstruction secondary to ureteral calculi. The authors concluded that MRU was able to diagnose acute ureteral obstruction secondary to ureteral calculi with similar accuracy to spiral CT. While ultrasound remains the initial diagnostic modality of choice in pregnant patients with suspected ureteral calculi, MRU appears to be an accurate alternative diagnostic tool in this patient population.
3. Spencer JA, Chahal R, Kelly A, Taylor K, Eardley I, Lloyd SN: Evaluation of painful hydronephrosis in pregnancy: magnetic resonance urographic patterns in physiological dilatation versus calculous obstruction. J Urol. 2004; 171: 256-60.

4. Regan F, Kuszyk B, Bohlman ME, Jackman S: Acute ureteric calculus obstruction: unenhanced spiral CT versus HASTE MR urography and abdominal radiograph. Br J Radiol. 2005; 78: 506-11.

Correspondence address: Dr. Brian R. Matlaga James Buchanan Brady Urological Institute The Johns Hopkins University School of Medicine 600 North Wolfe Street Baltimore, MD 21287, USA Fax: + 1410 502-7711 E-mail: bmatlaga@jhmi.edu 